\title{
A Patient With Progression of Diffuse Esophageal Spasm to Classic Achalasia
}

\author{
Seon Young Park ${ }^{*}$ and Jong Sun Rew \\ Department of Internal Medicine, Chonnam National University Medical School, Gwangju, Korea
}

A 58 year-old woman visited our department with a 2-month history of dysphagia on liquids and solids. Barium esophagogram showed pseudodiverticula suggesting simultaneous esophageal contractions and tapering at the gastroesophageal junction (Fig. 1). Esophageal manometry was performed in a standardized manner using an 8-channel water-perfused manometry catheter

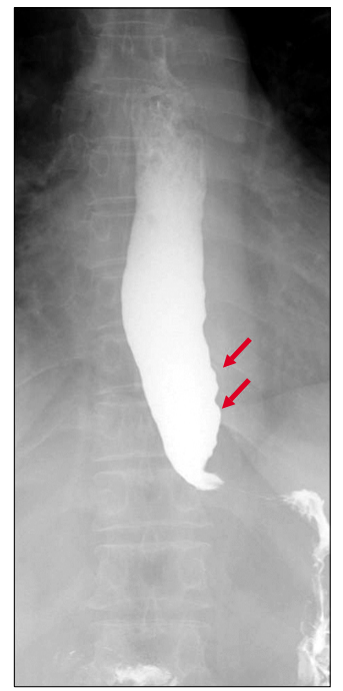

Figure 1. Barium esophagogram shows pseudodiverticula suggesting simultaneous esophageal contractions and tapering at the gastroesophageal junction.
(Synectics Medtronics, Stockholm, Sweden). We diagnosed her with diffuse esophageal spasm (DES) because the manometric features showed simultaneous contractions associated with $>$ $10 \%$ of wet swallows, mean simultaneous contraction amplitude of $>30 \mathrm{mmHg}$ and multiple peak contraction (Fig. 2A). ${ }^{1}$ She was advised to take diltiazem $30 \mathrm{mg} 3$ times a day and her symptoms improved. However, this motility disorder progressed to classic achalasia within 8 months (Fig. 2B). After pneumatic balloon dilatation, her dysphagia improved.

Several studies suggest that the motility disorder such as DES, nutcracker esophagus and achalasia share a common pathophysiologic mechanism, which involves the alteration in nitric oxide synthesis/degradation or loss of nitric oxide containing inhibitory neurons in the lower esophageal sphincter. The loss of intramural inhibitory neurons leads to the loss of normal peristalsis and inability of the lower esophageal sphincter to relax properly during swallowing. ${ }^{2-4}$ Some reports support the notion of progression of DES to achalasia. However, recent prospective cohort study showed that progression from DES to achalasia was uncommon (8\%) and did not identify predictors of progression to achalasia based on the initial manometry parameters. ${ }^{5}$ So, longer follow-up diagnostic tool such as high resolution manometry may help assess more accurately the pathophysiologic mechanism and true rate of progression from DES to achalasia.

Received: December 8, 2011 Revised: December 23, 2011 Accepted: December 29, 2011

(c) This is an Open Access article distributed under the terms of the Creative Commons Attribution Non-Commercial License (http://creativecommons. org/licenses/by-nc/3.0) which permits unrestricted non-commercial use, distribution, and reproduction in any medium, provided the original work is properly cited.

*Correspondence: Seon Young Park, MD

Department of Internal Medicine, Chonnam National University Medical School, 42 Jaebong-ro, Dong-gu, Gwangju 501-757, Korea

Tel: +82-62-220-6296, Fax: +82-62-228-1330, E-mail: drpsy@naver.com

Financial support: None.

Conflicts of interest: None. 
A

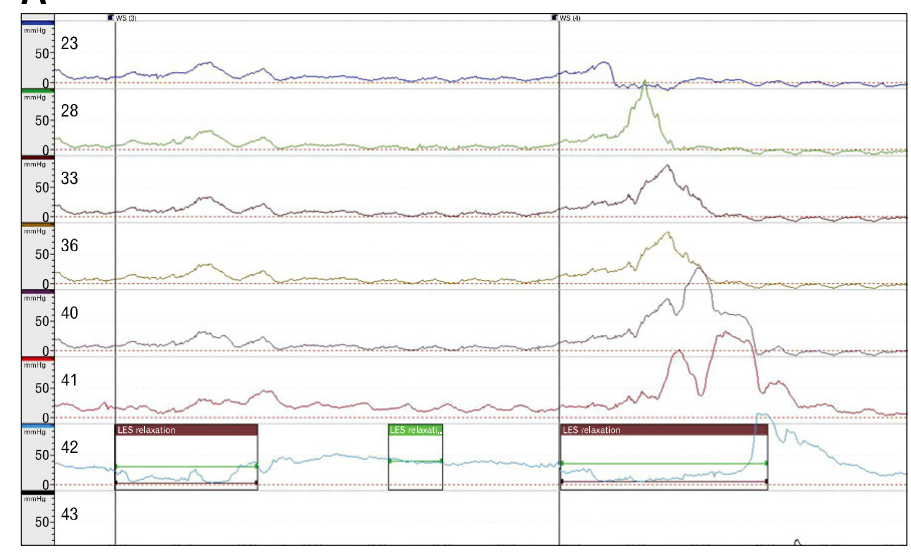

B

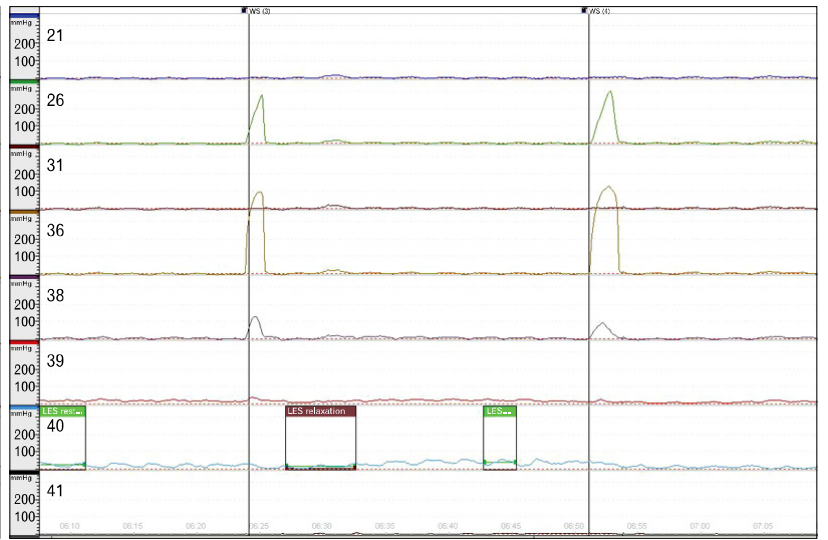

Figure 2. Esophageal manometry. (A) Manometric findings show simultaneous contractions associated with $>10 \%$ of wet swallows, mean simultaneous contraction amplitude of $>30 \mathrm{mmHg}$ and multiple peaked contractions. (B) After 8 months, follow-up manometric features show that lower esophageal sphincter relaxation become incomplete and contractions become to have low contraction amplitudes, which are the typical findings of classic achalasia. LES, lower esophageal sphincter.

\section{References}

1. Spechler SJ, Castell DO. Classification of oesophageal motility abnormalities. Gut 2001;49:145-151.

2. Sifrim D, Fornari F. Non-achalasic motor disorders of the oesophagus. Best Pract Res Clin Gastroenterol 2007;21:575-593.

3. Tutuian R, Castell DO. Esophageal motility disorders (distal esoph- ageal spasm, nutcracker esophagus, and hypertensive lower esophageal sphincter): modern management. Curr Treat Options Gastroenterol 2006;9:283-294.

4. Lacy BE, Weiser K. Esophageal motility disorders: medical therapy. J Clin Gastroenterol 2008;42:652-658.

5. Khatami SS, Khandwala F, Shay SS, Vaezi MF. Does diffuse esophageal spasm progress to achalasia? A prospective cohort study. Dig Dis Sci 2005;50:1605-1610. 